\title{
THE USE OF SHELL ELEMENTS TO CAPTURE SAIL WRINKLES, AND THEIR INFLUENCE ON AERODYNAMIC LOADS
}

\author{
D. Trimarchi, S. R. Turnock and D. J. Taunton, School of Engineering Sciences, University of Southampton, UK \\ D. Chapelle, INRIA, MACS team, France
}

\section{SUMMARY}

Sails are an example of fluid structure interaction (FSI), where the structural deformation, as well as the fluid dynamics, needs to be taken into account in order to analyse performances. The emphasis of this paper is placed on developing techniques that can study the dynamic behaviour of sails. This is necessary, especially when approaching downwind sails, where the nature of the flow is subjected to large separations and unsteady phenomena such as vortex shedding. Preliminary studies are presented about bluff body type flows, representative of spinnaker flows, and about typical Gennaker sections. From a structural perspective, the fabric is modelled with shells, rather than membranes. This approach shows considerable advantages, since these will capture buckling related phenomena such as wrinkling, which have to be treated with additional models for membrane based analysis. It is found that the unsteadiness and the presence of wrinkles can significantly alter the sail force

\section{NOMENCLATURE}

u vectors as bold lowercase letters

M matrices as bold capital letters

$f \quad$ scalars as cursive letters

ALE Arbitrary lagrangian eulerian

CFD Computational fluid dynamics

CST Constrant strain triangles membranes

MITCnl Mixed interpolation tensorial components nonlinear shells

RANS Reynolds averaged Navier Stokes

$A_{P} \quad$ Projected area

$C d \quad$ Drag coefficient

$\mathrm{Cl} \quad$ Lift coefficient

Co Courant number

$D \quad$ Diameter of a circular section ( $\mathrm{m}$ )

$d t \quad$ Time step magnitude ( $\mathrm{s}$ )

$E \quad$ Young's modulus $\left(\mathrm{N} \mathrm{mm}^{-2}\right)$

$F_{i} \quad$ i-th component of a Force (N)

$g \quad$ Gravity acceleration

$G M_{T}$ Transversal metacentric height ( $\mathrm{m}$ )

Kts Knots, unit for speed

$P \quad$ Pressure $\left(\mathrm{N} \mathrm{m}^{-2}\right)$

$Q \quad$ Mass flow rate $\left(\mathrm{Kg} \mathrm{m}^{-3}\right)$

$\mathrm{Re}_{d} \quad$ Diameter based Reynolds Number

$R \quad$ Radius of a circular section ( $\mathrm{m}$ )

$t \quad$ Sail fabric thickness ( $\mathrm{mm}$ )

$T \quad$ Period (s )

$T_{n} \quad$ Natural period ( s )

U Velocity $\left(\mathrm{m} \mathrm{s}^{-1}\right)$

$\mathbf{x}_{n} \quad$ Displacement vactor at n-th iteration

$\dot{\mathbf{x}}_{n} \quad$ Velocity vector at $\mathrm{n}$-th iteration

$\ddot{\mathbf{x}}_{n} \quad$ Acceleration vector at $\mathrm{n}$-th iteration

$y^{+} \quad$ Dimensionless distance from the wall

$v \quad$ Kinematic viscosity $\left(\mathrm{N} \mathrm{s} \mathrm{m}^{-2}\right)$

$v_{S} \quad$ Poisson ratio for the sail fabric

$\theta \quad$ Angle ( deg )

$\rho_{a} \quad$ Density of air $\left(\mathrm{kg} \mathrm{m}^{-3}\right)$

$\rho_{s} \quad$ Density of the sail fabric $\left(\mathrm{kg} \mathrm{m}^{-3}\right)$

\section{INTRODUCTION}

Sails have been analysed within engineering for almost forty years [1], and the state of the art is still evolving and growing rapidly. Due to the complexity of the coupled fluid-structural behaviour, the problem is far from solved. Downwind sail, in particular, require analysis of the interaction between fluid loading and structural responses since the difference between design and flying shapes are higher than for upwind sails. This is due to the combined effects of the very light material used for the construction, and the complex type of constraints, in which the sail may be assumed fixed at two points, with a third point free to move on a spherical trajectory. Despite such considerations, many authors neglected sail structural responses in the past, or used experimentally acquired flying shapes, in order to reduce the impact of the structural deformation $[2,3]$.

For upwind sails potential flow based codes have proved remarkably robust in predicting sail performance $[4,5,6]$. For sail flow regimes with significant areas of flow separation accurate load prediction requires the applications of flow solvers based on the Navier-Stokes equations. For reasons of computational cost analyses based on Reynolds averaged Navier Stokes equations (RANS) have been extensively used in recent years only for both upwind and downwind sails [7, 8].

Realistic sail Fluid -Structural Interaction analysis have only been introduced in recent years. Sails as a structure have generally been modelled as linear shape function membranes [4, 5, 9]. These elements are attractive for their relative simplicity, but there has been limited investigation of their reliability, although comparisons have been made for analytical test cases, either two-dimensional or axi-symmetric. Examples can be found, where these elements produce incorrect results [10].

The aim of the work presented is to explore the benefits of using shell rather than membrane elements and to consider how localised structural deformations (wrinkles) can influence the overall flow regime and resultant sail drive force. 
In the present research sails are analysed using four node Mixed Tensorial Interpolated Component (MITC) shell elements $[11,12]$. Such elements are capable of capturing all phenomena involved in thin structure deformation, namely membrane shear and bending deformations. Results are then compared with those obtained with the membrane Constant Strain Triangles (CST) elements, which traditionally have been implemented in sail structural solvers $[4,5,9]$. The research is carried out using the open-source software OpenFOAM for the fluid analysis. For the structural analysis the open-source software MODULEF was used, with the MITCnl Shell module.

\section{FLUID INVESTIGATIONS}

\subsection{DESCRIPTION OF FLOW SOLVER}

OpenFOAM (Open Field Operation And Manipulation) is a $\mathrm{C}++$ object oriented programming toolbox for solving several continuum mechanics problems, including CFD. It is licensed under the GNU general public licence. OpenFOAM uses finite volumes, discretized with structured or unstructured grids, to solve systems of partial differential equations. The standard version comes with several solvers, but one of the major features of the software is the (relative) ease in creating or modifying custom solver applications. OpenFOAM syntax is based upon the equation mimicking, therefore the high level code syntax closely resembles the governing equation of the problem to solve, making the higher-level code easier to understand.

In the present research the pisoFoam solver was used for the time marching analysis of the flow field. This solver implements the 'Pressure Implicit with Splitting for Operators' (PISO) algorithm, which is an unsteady version of the SIMPLE Algorithm. This uses an iterative solution of uncoupled equations for the pressure and velocity terms, present in Navier Stokes Equations. Details about the SIMPLE algorithm can be found in [13], and about the PISO algorithms in [14]. The Shear Stress Transport (SST) turbulence closure model was chosen for this work as it has been adopted in a number of previous works on sail type flows [15, 9, 8]. Wall functions implemented in OpenFOAM (KqRWallFunction and nutWallFunction) were selected for the close to the wall region treatment. Unless otherwise stated the first wall cell was chosen to have a normal direction size with a $y^{+} \sim 100$.

\subsection{THE 2D FLUID ANALYSIS}

Downwind sails are drag devices, where a certain amount of lift generation is involved. Since they are bluff-type bodies, and since the external motions caused by the yacht being in a seaway, an unsteady flow type is expected. In particular, a universal flow representation for the case of a symmetric spinnaker in symmetric, or almost symmetric flow, can be approximated as in the Fig.1-a, where velocity contours are sketched. On the leeward side of the sail, a bluff flow type is generated. On the windward side, since the particular shape of such a device, the sail's stagnation point is not placed on the sail surface, but about $0.75 \mathrm{R}$ upstream. This is particularly clear by looking at the iso-velocity contour in Fig.1. The same phenomenon can be observed later in terms of pressure (Fig.3), the distribution of which remains constant in the inner part of the sail. The device can therefore be assumed as a thick type body, as it is illustrated by the dashed line in Fig.1-b.

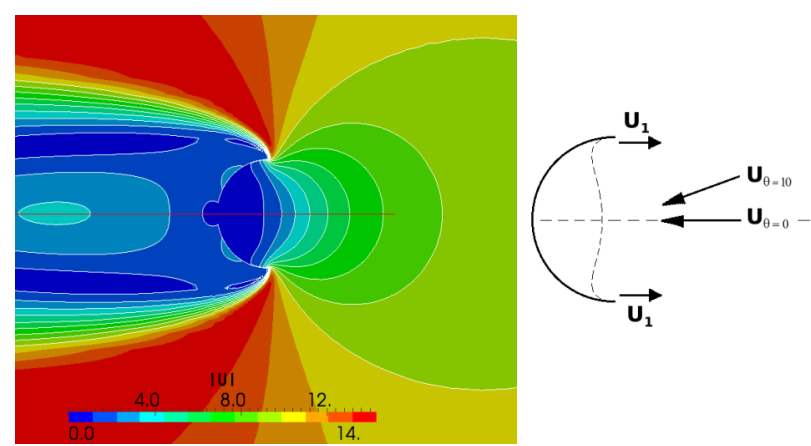

(a) Velocity isocontours (b) Effective sail shape Fig.1: Expected flow type for symmetric bluff bodies (flow right to left)

The flow developing on such device was analysed with two sets of simulations. In the first set, a zero degrees angle of attack was imposed, whereas in the second set of simulations the angle of attack was 10 degrees, as defined in Fig.1-b. An easy guideline for evaluating the symmetric case is the Euler's formula, where the force generated by the device is calculated via the mass flow rate and typical values are given in Table 1:

$$
F_{x}=\rho_{a} Q\left(\cos \theta \cdot U_{1}+U_{0}\right)
$$

Table 1. Typical values for bluff body flow including width based Reynolds number

\begin{tabular}{|c|c|c|c|c|c|}
\hline$\rho_{a}$ & $b$ & $U_{0}$ & $Q$ & $v$ & $\operatorname{Re}_{d}$ \\
\hline$\left[K g / m^{3}\right]$ & {$[m]$} & {$[m / s]$} & {$\left[m^{3} / s\right]$} & {$\left[m^{2} / s\right]$} & - \\
\hline 1.225 & 4 & 10 & 40 & $1.1510^{-5}$ & $3.4710^{6}$ \\
\hline
\end{tabular}

The value of $U_{1}$ was estimated from the CFD analysis, as $3.75 \mathrm{~m} / \mathrm{s}$, by taking the velocities average in the region of the border for $10 \%$ of the section width. With such values, the calculated force is equal to $250 \mathrm{~N}$ for the $2 \mathrm{D}$ case, which is 1.92 in terms of $C d$.

In the following, all forces are expressed in terms of nondimensional coefficients $C l=F_{y} /\left(0.5 \cdot \rho_{a} \cdot A_{P} \cdot v^{2}\right)$ and $C d=F_{x} /\left(0.5 \cdot \rho_{a} \cdot A_{P} \cdot v^{2}\right) . \quad F_{x}$ and $F_{y}$ are evaluated on a reference system, the $\mathrm{x}$-axis of which is directed along the direction of the flow. A structured mesh was chosen, in order to preserve central symmetry at all mesh points, preserving second order accuracy. A C-grid type grid was adopted for meshing the region close to the sail section. The mesh was designed with the open source software GMSH (http://geuz.org/gmsh/).

The domain was extended 5D upwind and 15D downwind in the stream direction, and 5D in the transverse direction, where $\mathrm{D}$ is the diameter of the 
chosen circular sail section. Uniform velocity and pressure were imposed at the inlet; uniform pressure and zero gradient velocity were imposed at the outlet. A constant slip velocity was imposed on upper and lower walls. KqRWallFunction and nutWallFunction were chosen for $\mathrm{k}$ and $v_{t}$. Freestream turbulence intensity was assumed $5 \%$. The initial value for $\mathrm{k}$ and $\omega$ were calculated as $k_{0}=3 / 2 \cdot\left(5 \% \cdot U_{I N}\right)^{2} ; \omega_{0}=\sqrt{k} / D$. SST-kOmega model was used, with Crank-Nicholson scheme in time $(\alpha=0.66)$. Three steps were imposed for the pressure correctors, and one for the non orthogonal corrector. The time step was chosen such as the maximum Courant number ( $\mathrm{Co}$ ) remain smaller than one. The Co is an indicator of the propagation of the numerical error within the domain, and it is a function of the calculation time step and the mesh size. The condition $C o<1$ assures that numerical errors are smoothed away, rather than propagated within the domain as the calculation advances.

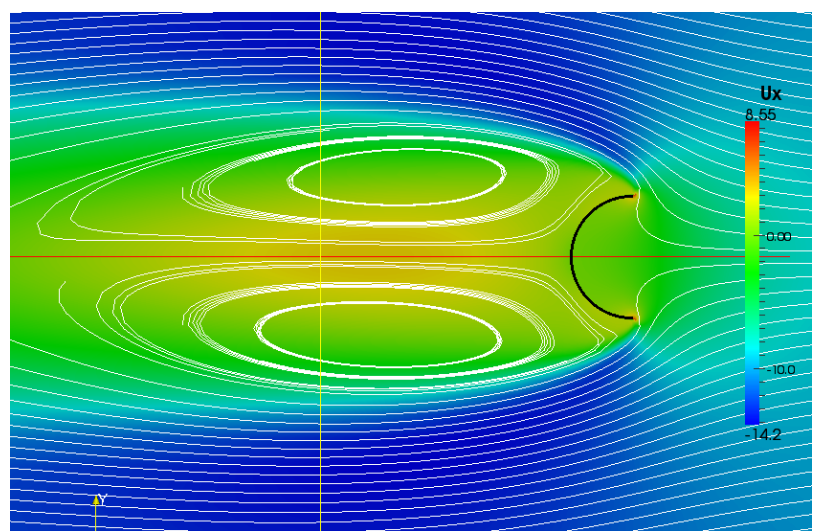

Fig.2: Symmetric attached flow, velocity and streamlines

In Fig.2 the field is visualized in terms of velocities and streamlines for a flow with zero angle of attack. In this case, the flow remains steady and two symmetric vortices are formed behind the sail. However, when the flow rotates, the introduction of an angle of attack removes the symmetry. This produces a highly unsteady flow with periodic vortex shedding, as visualized in Fig.3, where the four images represent one cycle of vortex generation in terms of pressure.

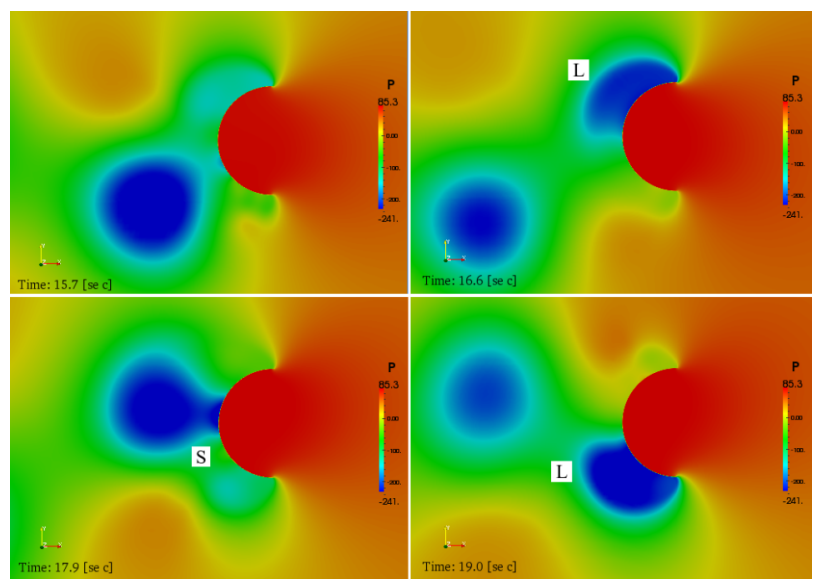

Fig.3: Vortex shedding generation cycle. St $\approx 0.5$
In Fig. 3 -a the vortex is located just left of the sail section on the left side. A vortex is then generated on the right side (Fig.3-b). This is underlined on the figure with the letter L, which stands for lower pressure vortex. Moving downstream, the vortex splits into a primary vortex, which continues downstream, and a secondary vortex, here marked with $\mathrm{S}$, which collapses back onto the sail's surface (Fg.3-c). A periodic system cycle is then closed with the formation of the last vortex on the left side of the sail section (Fig.3-d).

In terms of driving force generation, when the angle of attack is zero and the flow is steady, the value $-237 \mathrm{~N}$ is comparable with the estimate from the Euler's formula, and no transverse force is experienced (Fig.4). When introducing an angle of attack, the unsteadiness induced into the flow produces a higher force generation, where the average drag coefficient passes from 1.82 to 3.48 , with an increment of about $90 \%$. In the figure, markers L and $\mathrm{S}$ are reported, as in Fig. 3 .
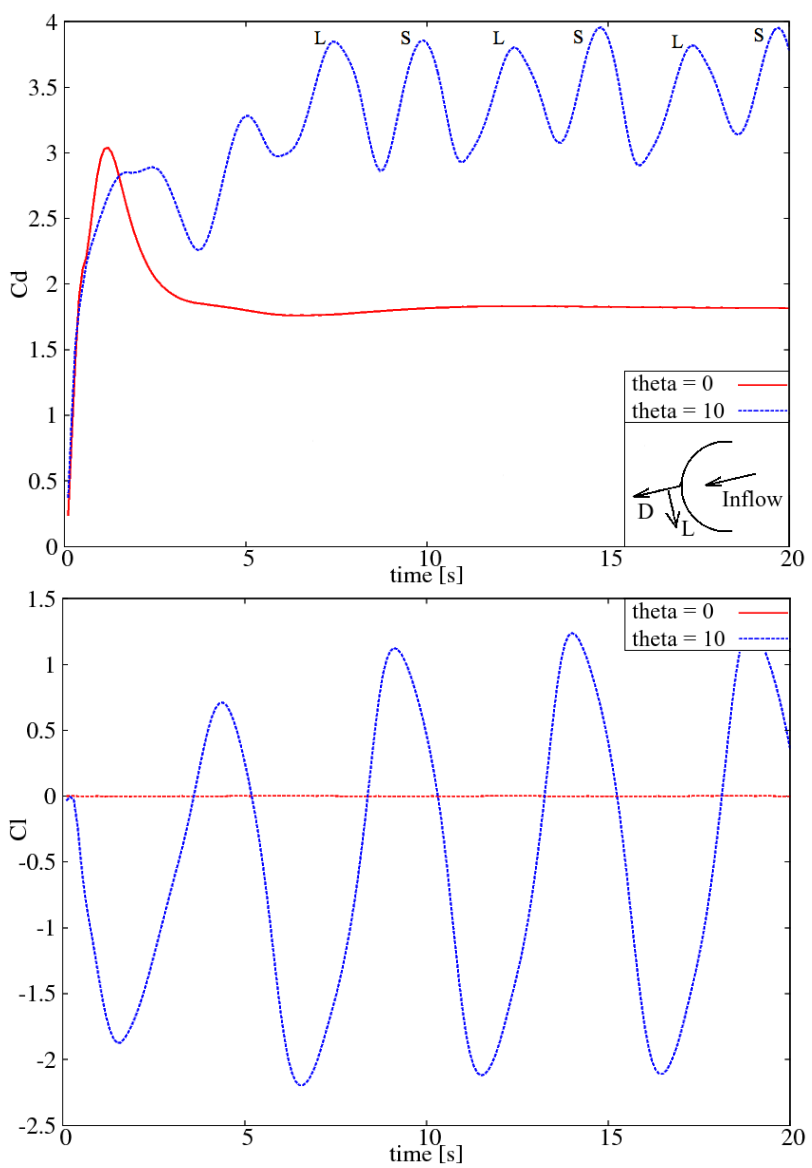

Fig.4: Steady/unsteady force comparison

The applicability of such results to the full three dimensional flow regime is very limited, because of the differences arising between two and three dimensional flow. Additionally, the unsteady RANS equations have known limitations in dealing with bluff body periodic separated flow [16]. Four meshes were used for the mesh sensitivity study, the characteristics of which are summarized in terms of time averaged quantities in Table 2. Such values have been estimated by time averaging the force history, which is reported in Fig.5. A 
considerable amount of mesh sensitivity is therefore experienced, and this affects both the magnitude of the force and its oscillation period.

However, the average drag force tends to stabilize quite well, once the $y^{+}$value assumes reasonable values.

Table 2

\begin{tabular}{|l|l|c|c|c|c|c|}
\hline Mesh & N. Hexa & $y^{+}$ & $C d$ & $C l$ & $T[s]$ & $\mathrm{dt}[\mathrm{s}]$ \\
\hline Coarse & 3774 & 1500 & 3,140 & -0.016 & 4.6 & $510^{-3}$ \\
\hline Base & 19245 & 300 & 3.300 & 0,135 & 5 & $10^{-3}$ \\
\hline Fine & 45594 & 200 & 3.266 & -0.231 & 5.5 & $710^{-4}$ \\
\hline Finer & 121519 & 100 & 3.277 & -0.213 & 5.2 & $510^{-4}$ \\
\hline
\end{tabular}
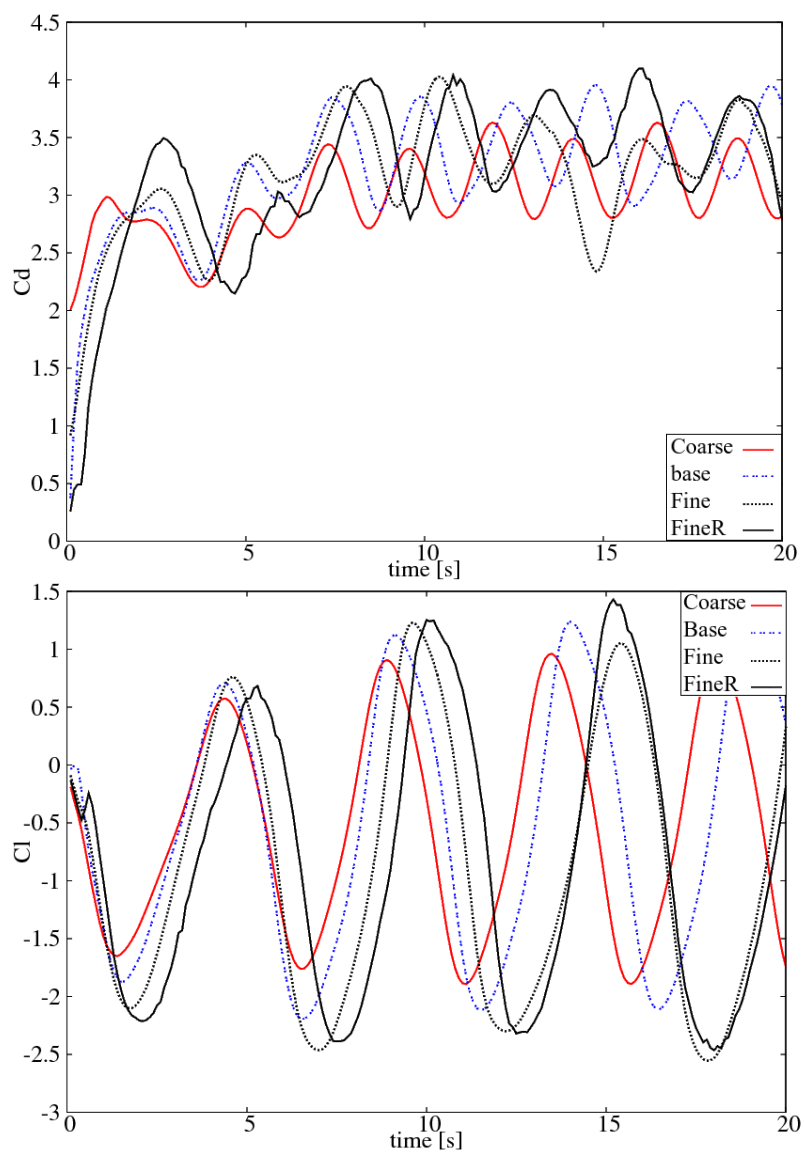

Fig.5: Mesh sensitivity in terms of force time history

Although lift (sideforce) is generated, its averaged value has a strong mesh sensitivity. It should be remarked in fact that the value of $\mathrm{Cl}$ calculated on the 'Base' mesh only is positive, where it goes negative for all other meshes. The lift generation process is then quite unstable in this situation. The conclusion is not surprising, since on such a device the generated lift can be estimated as a second order process.

The vortex shedding oscillation period was estimated by evaluating the time distance between two higher peaks of the time signal. Its value seems to increase as the mesh become finer, but its value seems to converge quite well.

In the following, the geometry has been updated in order to analyse a much more realistic sail section. A typical Gennaker section was then derived by [17]. The angle of attack was imposed $22.5^{\circ}$, which is the angle of maximum lift, as shown in [17]. The new mesh was designed in order for the average $y^{+}$to be around 100 . A set of analysis was performed, with the objective of investigating the influence of the velocity in the force generation process. Five different inlet velocities were imposed, as summarized in the Table3:

Table 3

\begin{tabular}{|l|l|l|l|l|l|}
\hline $\mathrm{U}\left[\mathrm{m} \mathrm{s}^{-1}\right]$ & 2 & 3.5 & 5 & 7 & 10 \\
\hline $\operatorname{Re}_{d}$ & $6.910^{6}$ & $1.210^{7}$ & $1.710^{7}$ & $2.410^{7}$ & $3.510^{7}$ \\
\hline
\end{tabular}

The flow field is represented in Fig. 6 in terms of velocity magnitude and streamlines. The flow in this case does not produce vortex shedding, and remains steady. A separation bubble is generated at the leading edge, leeward side, by the sharp edge. Some recirculation can also be identified in the large separated region, the development of which is driven by the adverse pressure gradient on the second half of the sail leeward.

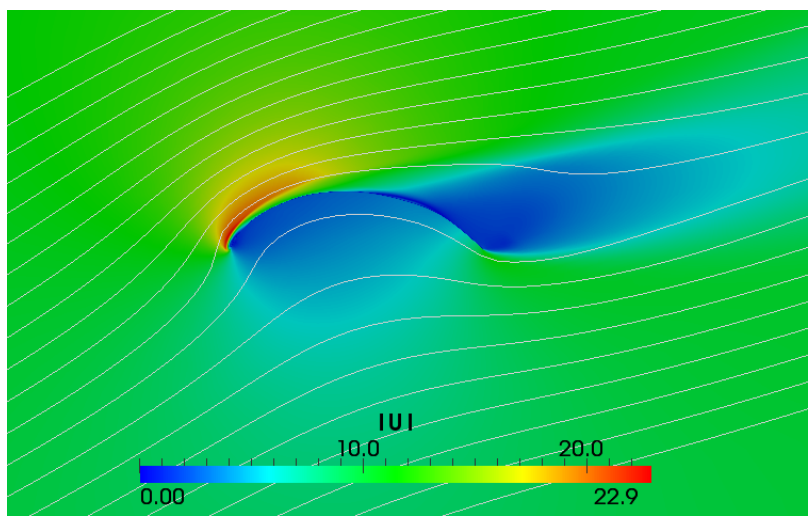

Fig.6: The flow on a typical Gennaker section

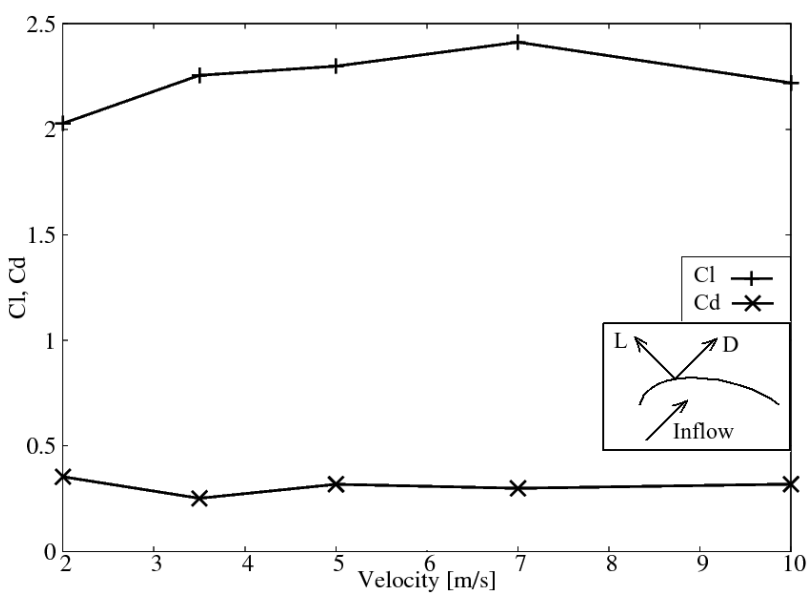

Fig.7: Force coefficients over velocity

Results in terms of force coefficients are reported in Fig. 7 in terms of lift and drag coefficients versus inlet velocity. No strong sensitivity was experienced. However, for the slower $\left(2 \mathrm{~m} \mathrm{~s}^{-1}\right)$ and for the faster $(10 \mathrm{~m}$ $\mathrm{s}^{-1}$ ) case some instability was encountered during the calculation. For the lower speed, the explanation could be in the mesh definition, since the mesh was calibrated for the higher speed regime. When performing the lower 
speed analysis, the average $y^{+}$drops to 27.7 , which is close to the limit of the validity range for using wall functions.

For the higher speed, the instability could be related to a difficulty to capture the separation point, when a recirculation zone starts to develop in the separated region. Capturing the separation point due to an adverse pressure gradient is a known weakness of RANS. This approach tends in fact to 'freeze' the unsteady flow features, thus slightly under-predicting the separation region length.

\subsection{THE 3D FLUID ANALYSIS}

Analysis similar to those obtained in two dimensions have been performed for the three dimensional case. The mesh was in this case derived from the two-dimensional mesh, by extruding the lower sections and adding a spherical dome at the top of the device. In Fig.8 the sail surface and the surrounding C-grid mesh structure are shown. 1.6 milion elements were used for the entire domain discretization, and a time-step of $0.002 \mathrm{~s}$. was chosen, in order for the Courant Number to remain lower than 1 . The field was initialised as previously, with an angle of attack of 10 degrees. All relevant calculation parameters were left unchanged, as in the twodimensional case. The case was run on the University of Southampton cluster IRIDIS3. The parallel features of OpenFOAM were used. The case was therefore decomposed in 24 domains, running on three eightprocessors nodes. The solution was obtained in about 10 sec for every time-step, and a global solution, resolving the flow for 35 [sec] was obtained in about 48 hours.

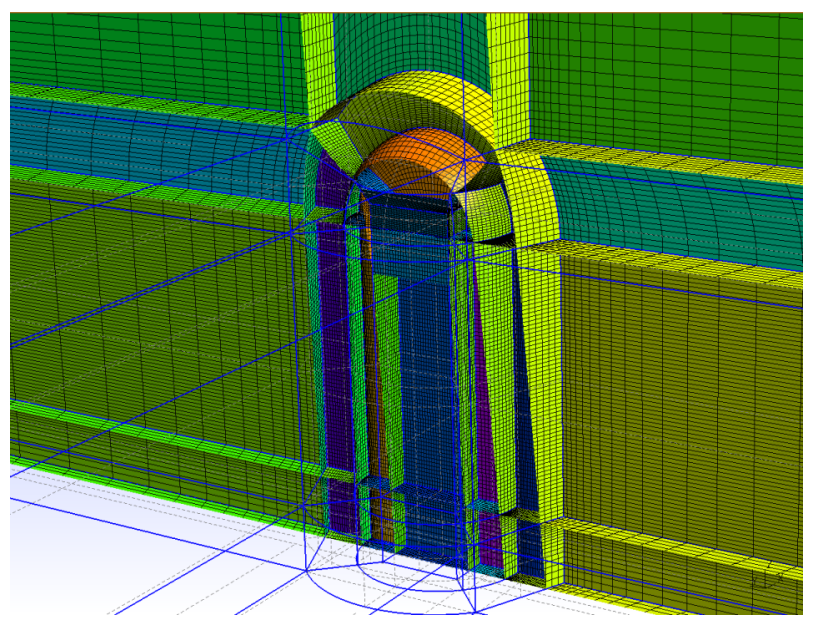

Fig.8: Three dimensional mesh structure

overview of the flow regime is shown in Fig.9, where three orders of streamlines are reported, and the colormap represents the pressure. A very large separation zone can be identified behind the sail, where the flow behaviour seems quite chaotic and it is not possible to identify any defined flow structures. This higher degree of flow mixing causes the flow to remain steady, as it is clear in Fig.10, where forces are reported in time.

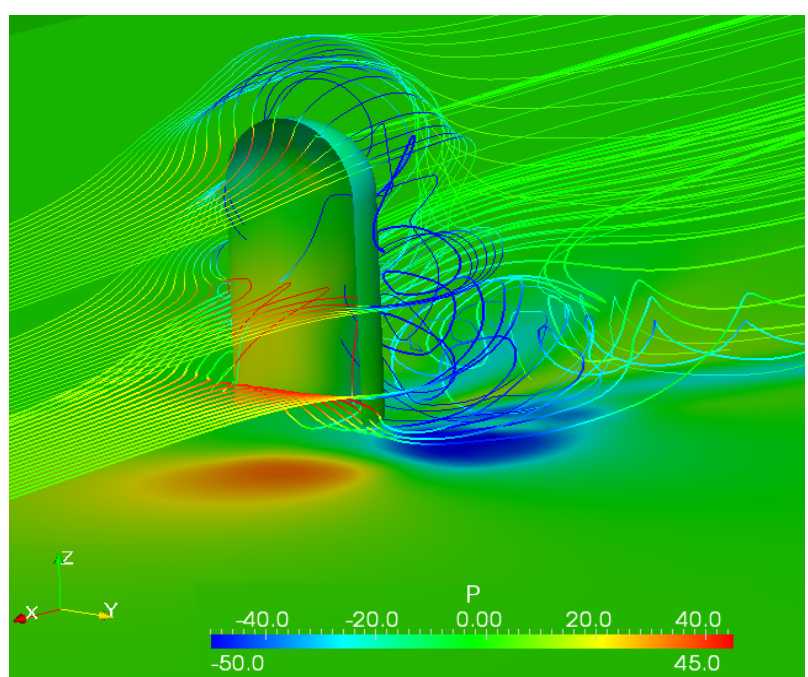

Fig.9: Three dimensional analysis results

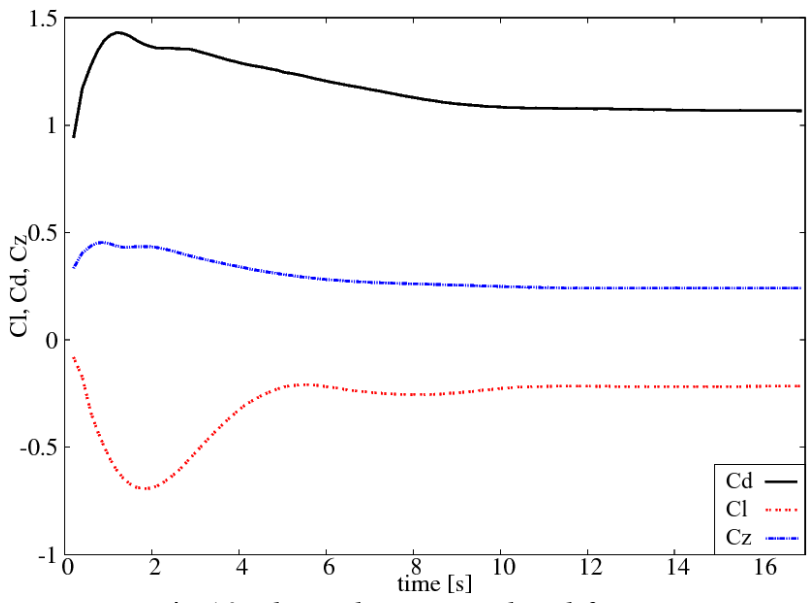

Fig.10 Three-dimensional sail force generation

The question arises then whether an induced unsteadiness, caused by the boat's motions, can induce some additional drag. The three dimensional analysis was then carried on by imposing unsteady boundary conditions at the domain inlet. It was chosen to investigate a variation in the angle of attack, by leaving all other relevant parameters unchanged.

It can be shown that a boat's rolling period can be estimated as:

$$
\begin{gathered}
T_{n}=\frac{(\alpha \cdot B)}{\sqrt{\left(g \cdot G M_{T}\right)}} \approx 5.4[\mathrm{sec}] \\
\text { where }: \alpha \approx 5.5 \text { for a sailboat } \\
B_{Y D 40}=3.7[\mathrm{~m}] \text { boat' } \mathrm{s} \text { beam } \\
\left.\quad G M_{T}\right|_{Y D 40}=1.452[\mathrm{~m}]
\end{gathered}
$$

A rotating velocity was then imposed, varying angle of attack with a symmetrical oscillation of 5 degrees around the initial value. The inlet condition was then imposed in order for the angle of attack to vary between 5 to 15 degrees with a $5.4 \mathrm{~s}$ period. Results from this analysis are reported in Fig.11, where the drag behaviour and the angle of attack variation are plotted against time. 4 cycles were imposed, in order to get a fully developed periodic behaviour. The average value of the unsteady induced 
drag coefficient can be estimated as 1.143, whereas the steady drag coefficient is about 1.09. Compared to the steady case, the induced unsteadiness produces an increase of about $5 \%$ in the average generated drag.

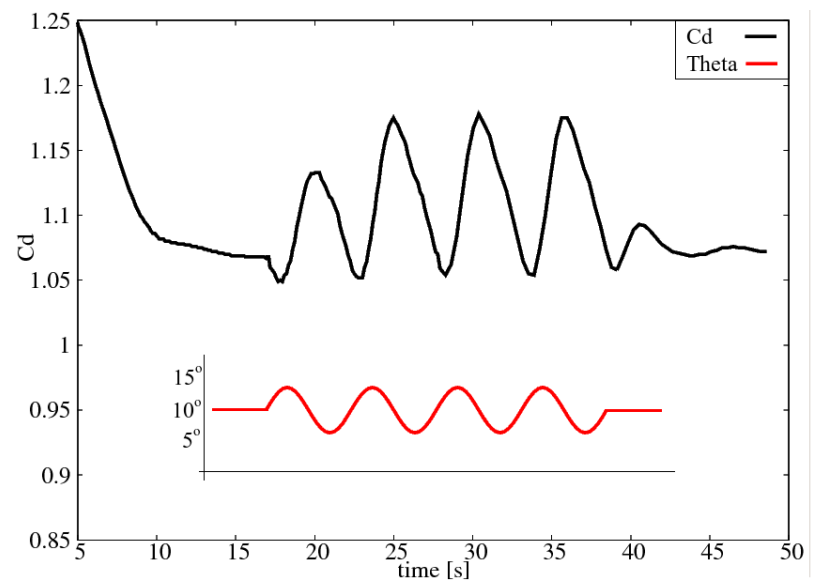

Fig.11 Impact of induced unsteadiness on drag

\section{STRUCTURAL CALCULATIONS}

\section{$3.1 \quad$ MODULEF AND MITCnl DESCRIPTION}

Modulef is an open source Finite Element library, which contains about 3000 procedures written in Fortran 77. The French research centre INRIA developed the software. Many operators are already present in the MODULEF library and new mathematical operators are incorporated into the library as computational modules. More details can be found online, at the address: http://www-rocq.inria.fr/modulef/

Within the MODULEF environment, the finite element package MITCNL is implemented as a module. This is a set of finite element subroutines, which implement the element-wise computations (stiffness matrices, load vectors, stresses...) for the MITC shell non-linear elements. The MITC elements - standing for "Mixed Interpolation of Tensorial Components" - are particularly effective and robust elements of the "general shell element" family [12].

The main difference between shell and membrane elements is in the number of degrees of freedom per node. Membranes consider 3 degrees of freedom per node, namely translations in the space, but bending stiffness is completely neglected. Shells have 5 degrees of freedom per node, the displacements and the rotations around the tangent axis, and the rotation around the normal axis is neglected. The mid surface describes the shell geometry, the curvature of which is governed by the normal vector at nodes. The shell geometry is represented by the three dimensional chart: $\vec{\Phi}\left(\xi^{1}, \xi^{2}, \xi^{3}\right)=\vec{\phi}\left(\xi^{1}, \xi^{2}\right)+\xi^{3} \cdot \vec{a}_{3}\left(\xi^{1}, \xi^{2}\right)$, where $\vec{\phi}\left(\xi^{1}, \xi^{2}\right)$ represents the shell mid surface, and $\xi^{3} \cdot \vec{a}_{3}\left(\xi^{1}, \xi^{2}\right)$ the normal extrusion at the point of coordinates $\left(\xi^{1}, \xi^{2}\right)$, and $\left.\xi^{3} \in\right]-t\left(\xi^{1}, \xi^{2}\right) / 2, t\left(\xi^{1}, \xi^{2}\right) / 2[$.
The mixed interpolation, which the name MITC refers to, is crucial in order to avoid Locking. This is a well-known phenomenon, which affect the shell's structural response when thickness goes to zero, like for instance in the case of sail fabrics. Although sails have traditionally been analysed with membrane elements, shell finite elements have been chosen for the research, since they are suitable to reproduce all phenomena involved in sail deformation analysis. With membranes, important deformation components are neglected, as in particular buckling related phenomena such wrinkling. This will be discussed in the following sections.

Although the MITCnl Package is not open source, the source code was made available to the author thanks to an agreement signed between the French research centre INRIA and the University of Southampton.

\subsection{STRUCTURAL ANALYSIS DESCRIPTION}

A dynamic calculation has been implemented for the sail's analysis, for two principal reasons. From a numerical point of view, adding mass and damping terms helps convergence in the first time steps. Due to the very limited thickness in fact, analysis of such structures is very sensitive to any small perturbation, resulting in large displacements, and a highly ill conditioned system. A dynamic calculation will then be necessary in the future, when performing unsteady FSI.

The system to be solved is then:

$$
\mathbf{M} \cdot \ddot{\mathbf{x}}+\mathbf{C} \cdot \dot{\mathbf{x}}+\mathbf{K}(\mathbf{x}) \cdot \mathbf{x}=\mathbf{F}(t)
$$

Where:

M Mass matrix, defined as: $\int \rho_{s} \cdot \mathbf{P} \cdot \mathbf{P}^{T}$

P Shape function polynomial

C Damping matrix, to be defined in the following section

K Stiffness matrix, defined as: $\int B^{T} \cdot D \cdot B$

B Matrix of derivatives

D Constitutive relationship matrix

By discretization:

$$
\begin{gathered}
\ddot{\mathbf{x}}=\frac{\dot{\mathbf{x}}_{n+1}-\dot{\mathbf{x}}_{n}}{d t} ; \quad \dot{\mathbf{x}}=\frac{\mathbf{x}_{n+1}-\mathbf{x}_{n}}{d t} ; \quad \mathbf{x}=\mathbf{x}_{n+1 / 2} \\
\frac{\dot{\mathbf{x}}_{n+1}+\dot{\mathbf{x}}_{n}}{2}=\frac{\mathbf{x}_{n+1}-\mathbf{x}_{n}}{d t}=\frac{2 \cdot\left(\mathbf{x}_{n+1 / 2}-\mathbf{x}_{n}\right)}{d t}
\end{gathered}
$$

The governing equation becomes then:

$$
\begin{aligned}
\left(\frac{4 \mathbf{M}}{d t^{2}}+\frac{2 \mathbf{C}}{d t}+\mathbf{K}\right) \cdot \mathbf{x}_{n+1 / 2}=\left(\frac{4 \mathbf{M}}{d t^{2}}+\frac{2 \mathbf{C}}{d t}\right) \cdot \mathbf{x}_{n}+ & \frac{2 \mathbf{M}}{d t} \cdot \dot{\mathbf{x}}_{n}+ \\
& +\mathbf{F}_{n+1 / 2}
\end{aligned}
$$

The algorithm, implemented within the MODULEF program, is detailed in Fig. 12. 


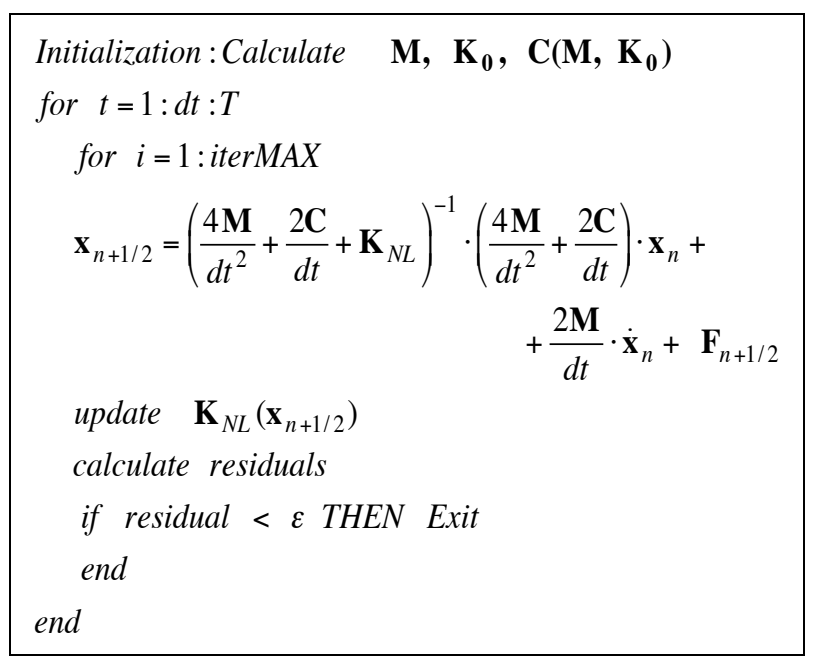

Fig.12 Calculation flow chart

The residual is calculated evaluating the $L^{2}-$ norm of the displacement vector at $i-t h$ and $(i-1)-t h$ iteration.

The sail has been discretized with 4 node MITCnl elements. The sail used for the analysis is designed as a cylinder body, over which a spherical sector is posed, as is Fig.13. Singularities in the imposition of boundary conditions are avoided by cutting a circular section in correspondence to the sail's corners. The Sail's height is $3000 \mathrm{~mm}$, and the circular radius is $1000 \mathrm{~mm}$. A simple support has been imposed on the sail corners, whereas symmetry condition has been imposed on the sail centre. Four different meshes have been tested for the sensitivity study. The coarser mesh has 20 elements per horizontal section, and the finer 80 .
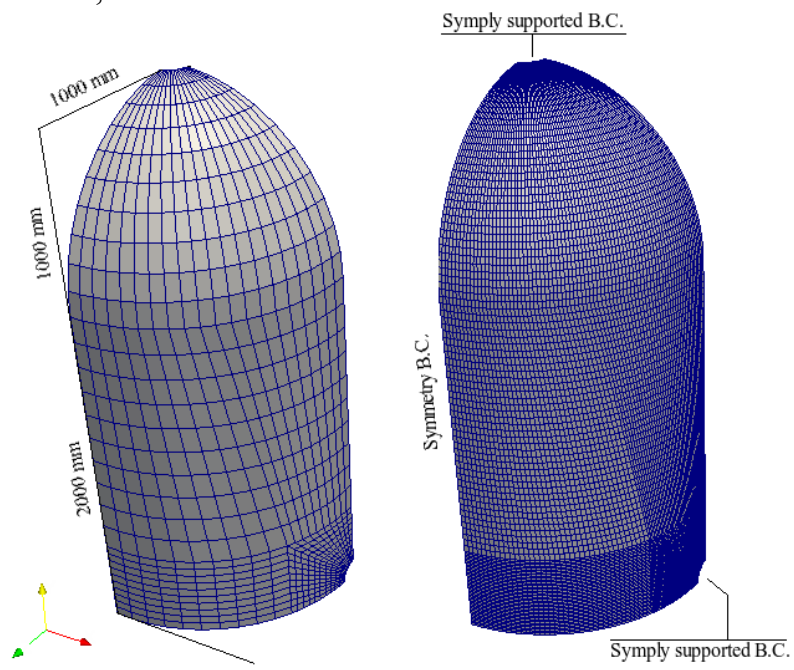

Fig.13 Meshes for structural calculations

The material has been considered as isotropic. The characteristic constants and the applied loading are defined in the following table:

Table 4

\begin{tabular}{|c|c|c|c|c|}
\hline $\mathrm{t}$ & $\mathrm{E}$ & $\rho_{s}$ & $v_{s}$ & $\mathrm{P}$ \\
\hline $\mathrm{mm}$ & $\mathrm{N} / \mathrm{mm}^{2}$ & $\mathrm{Kg} / \mathrm{mm}^{3}$ & - & $\mathrm{N} / \mathrm{mm}^{2}$ \\
\hline 0.05 & $3.67 \cdot 10^{2}$ & $1.15 \cdot 10^{-6}$ & 0.3 & $4 \cdot 10^{-4}$ \\
\hline
\end{tabular}

The load is constituted by a prescribed surface distributed force, represented as a series of concentrated loads on the element's nodes. The load intensity corresponds to an apparent wind speed of about $8 \mathrm{~m} / \mathrm{s}$, e.g. 15 Kts.

Rayleigh Damping was chosen for the definition of the Damping Matrix. This matrix in fact can not be assembled from element damping matrices, as in the case of stiffness and mass matrices. The damping matrix is therefore calculated as a linear combination of Mass and Stiffness matrices:

$$
\mathbf{C}=c_{1} \cdot \mathbf{M}+c_{2} \cdot \mathbf{K}
$$

Details of this procedure can be found in [11].

It can be shown that the global damping coefficient of the system can be written as:

$$
\xi=\left(c_{1}+c_{2} \cdot \omega^{2}\right) /(2 \cdot \omega)
$$

Good values for an effective damping are calculated then for $c_{1}=\omega_{n} ; c_{2}=1 / \omega_{n}$, which assures a critical damping $(\xi=1)$ for the structure's natural frequency, and an over-damping on higher frequencies.

The natural frequency $\omega_{n}$ was calculated exporting the linear stiffness matrix in Matlab, and solving the eigenproblem $\mathbf{K} \cdot \mathbf{x}=\omega^{2} \cdot \mathbf{M} \cdot \mathbf{x}$

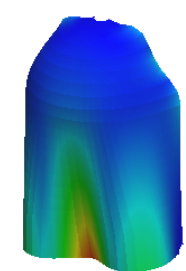

Mode 1

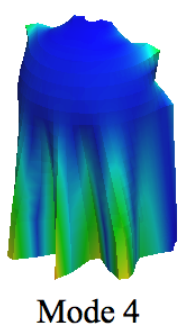

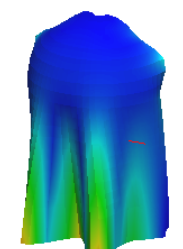

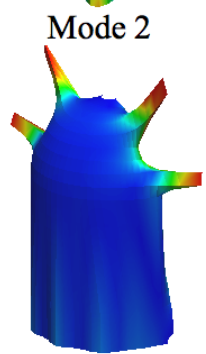

Mode 5

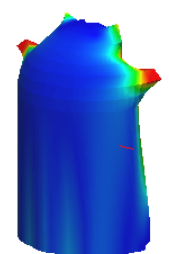

Mode 3

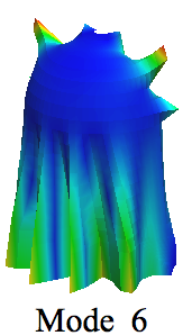

Fig.14 Eigenvectors calculated for the spinnaker

Although only the first natural frequency was of interest, corresponding to the first Eigenvalue, ten Eigen modes were calculated, in order to have a more detailed idea of the harmonic behaviour of the structure. For the $10^{\text {th }}$ eigenvector, 10 oscillations per section can be expected, the description of which requires about 20 elements per section. That's why a coarse mesh with 20 elements per horizontal section was chosen for the Eigen-analysis. The linear initial stiffness matrix was used, e.g. the stiffness matrix calculated for zero displacement. As a result, a number of Eigenvalues and Eigenvectors were calculated, representative of the structural vibration modes and corresponding frequencies. Since the calculation was performed with the linear stiffness matrix, the value of the natural frequency at this stage is not representative of the nonlinear physics of shell deformation, but an artificial value 
used for calculating an effective damping matrix, therefore facilitating the convergence. In particular, the structure's natural frequency is in this stage very small $(0.0073 \mathrm{~Hz})$, corresponding to a very high natural period (860 s). The actual natural frequency, calculated at the equilibrium with the non-linear stiffness, will be higher. This means that such coefficients produces an overdamped (and therefore slower in terms of convergence) analysis. This is the well known behaviour for a simple harmonic oscillator. In the case of zero damping, the structure continues to oscillate. When the damping coefficient value is $0<\xi<1$ oscillations are progressively damped. Critical damping arises for damping coefficient $\xi=1$. In that case no oscillations are experienced and the structure converges straight to the steady final position. For over-damping, when $\xi>1$, no oscillations are experienced, but a longer time is needed to reach the final equilibrium position.

In Fig. 14 the firsts 6 Eigenmodes are sketched. It is interesting to remark that peaks are arising on the free sides, particularly evident for Eigenmodes 3,5 and 6 . This corresponds to a locally ill posed problem, which could cause instabilities in the calculation. 20 time steps per natural frequency were judged a good value for the analysis, this leading to a time step $\mathrm{dt}=42 \mathrm{~s}$ for the linear analysis.

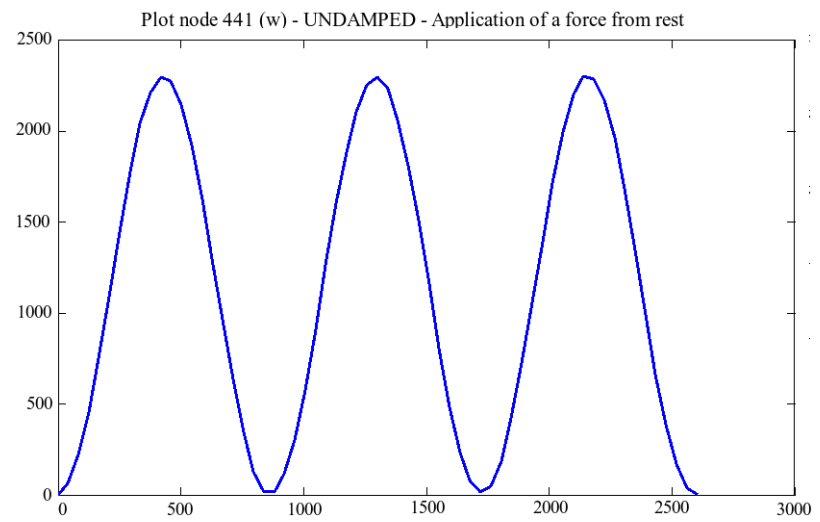

Fig.15 Linear calculation - Application of a force from rest - No Damping

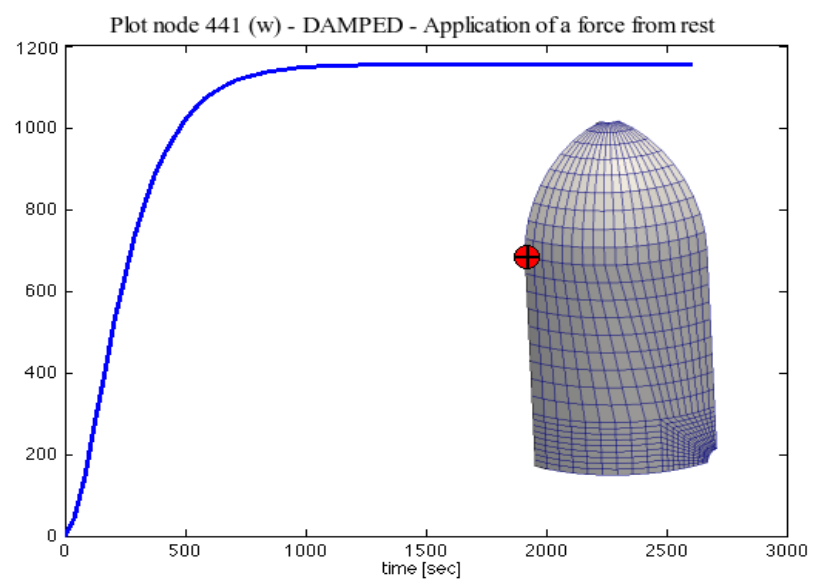

Fig.16 Linear calculation - Application of a force from rest-Damping allowed
In Fig.15-16 the horizontal displacements of a node placed on the sail's symmetry line is plotted versus time, for the linear calculation. A constant horizontal force constitutes the load. This test has been introduced for studying the structural behaviour in a simplified case and verifies that the analysis is consistent, before moving to non-linear calculations. In the first figure, damping is not allowed. Therefore the structure oscillates at the natural period. In the second figure damping has been introduced, therefore no oscillation is present, and convergence is achieved in the time of a natural oscillation.

When moving to the non-linear analysis, convergence becomes more difficult. This happens since the tangent stiffness matrix $\mathbf{K}_{N L}=\mathbf{K}_{0}+\mathbf{K}_{N L}(\mathbf{x})$ changes dramatically, and at a very high rate, in the first time steps. This leads to some oscillations, resulting in the need for a very small initial time step to avoid divergence. However, after the initial oscillations the stiffness matrix becomes more stable, allowing much higher time steps. A variable time step has therefore been imposed, increasing 10 times every 50 time steps.

In Fig.17, the displacement of a symmetry point is plotted over time.

It clearly visible that in the first part of the graph (0-100 s) oscillations arise, caused by the non-linear stiffness matrix, which evolves at a very high rate in this phase of the calculation. However, once the first part of the nodal path is overcome, convergence become smooth, and an over-damped type behaviour can be recognized.

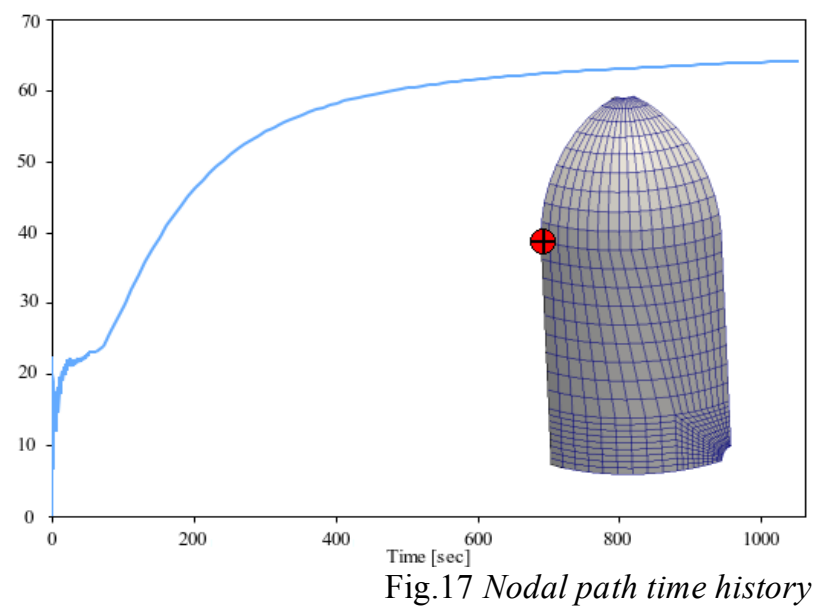

Such results has been compared with those calculated with SailFEM, a Finite Element code developed in Matlab for dealing with the CST membrane elements proposed by [18]. Details about the actual implementation of the code can be found in [19].

The comparison is performed on similar mesh size, but it is worth remarking that elements implemented in SailFEM are triangular (CST), whereas shells MITCnl are quadrilateral. The same characteristic material properties, loadings and boundary conditions were applied to the two calculations. In Fig. 18 the results of this comparison are shown, where the colourmap represents the norm of nodal displacements. The 
calculated shape is quite different, and the membrane CST looks very 'stiff' compared to shell MITCnl elements. However, the order of magnitude of global displacements is the same in the two analyses.

In the higher part of the sail, some wrinkles are identified by the shell calculation. This will be discussed in the following section. However, it is worth remarking that some oscillations are visible also in the membrane deformed shape. Such oscillation should not be interpreted as wrinkles, since the membrane model is unable to represent such phenomena. Such instabilities are to be interpreted as a numerical error due to the very coarse mesh adopted for the calculation.

In Fig.19 the two deformed shapes are sketched, where results obtained with SailFEM are represented in yellow, and MODULEF results are represented in red. From this comparison it is even clear that the two answers are of the same order of magnitude, but the distribution of displacements in the deformed shape has changed slightly.

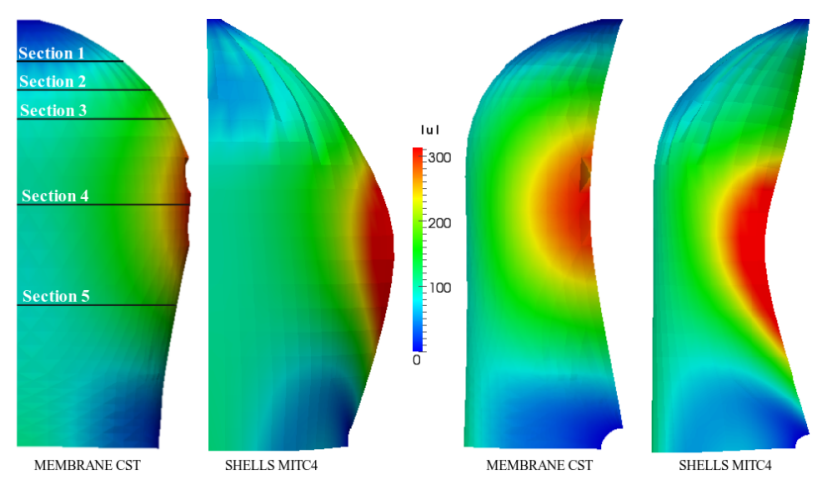

Fig.18 Results obtained with CST membranes and MITCnl shells in terms of nodal displacements

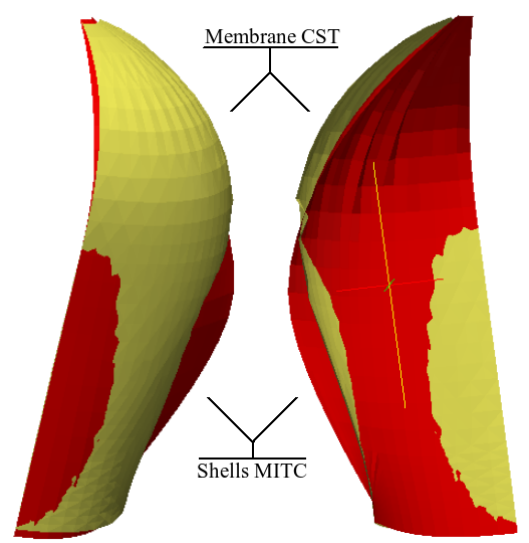

Fig.19 Comparison between MODULEF and SailFEM

The four sections identified in the three dimensional plot in Fig.18 are drawn in Fig.20, where four sail sections are compared against the initial un-deformed section shape.

In the higher part of the sail displacements are much higher for CST elements where the fabric looks much more stretched for the shell answer. In the lower third of the sail the behaviour predicted by the shell model is less stiff. This arises especially on the sail's trailing edge, where the angle of the fabric looks much more 'opened' compared to the membrane model. Some considerations on such behaviour will be addressed in the following section.

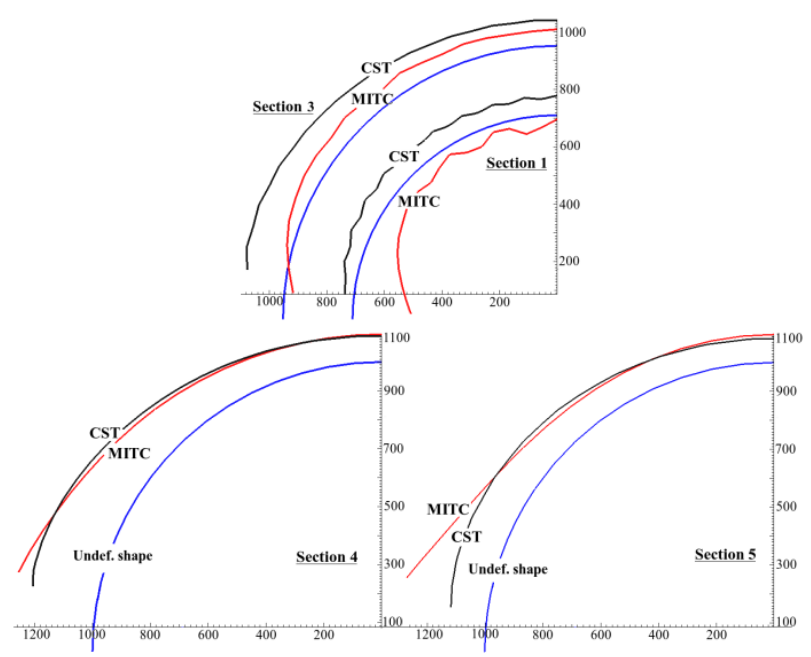

Fig.20 Sail sections comparison

Some considerations should be done about the instability arising on the spinnaker's free sides for the membrane calculations, as underlined in Fig.21. Such instability should not be thought of as a numerical error, but it resides in the physics of the problem itself. Looking at Fig.14, strong singularities had been identified with the eigenvectors in the spinnaker's head zone. Such instabilities correspond to a locally ill posed problem for the membrane model [12, Par 5.3], in which the bending energy must be taken into account. In such problems, the membrane deformation itself is unable to control the displacement field induced by the loading, indeed. The shell model is capable of controlling such deformation energy, since it includes the bending stiffness. However, due to the very limited thickness, the shell response still reflects the instabilities, albeit in a smoother form.

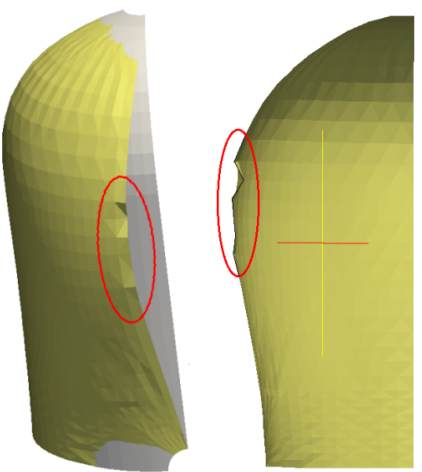

Fig.21 Instability arising for the membrane problem

\subsection{WRINKLING GENERATION}

Sails are subjected to local buckling related phenomena, known as wrinkling. Taking into account these phenomena is important, since they can modify the stress distribution within the structure, resulting in local uniaxial stresses distributions. From an energy point of view the membrane behaviour is in fact insufficient for 
reproducing the development of wrinkling, which is driven by the (very low) sailcloth bending stiffness, and occurs when the membrane stresses cannot be selfbalanced.

It can be observed that the wrinkles do not necessarily follow the sailcloth seams. Therefore, the assumption of isotropic homogeneous material is not affecting the capability of shells elements to reproduce wrinkles.

In the literature ad hoc models have been proposed, which locally change the constitutive relationship of the membrane model in zones where a wrinkling criterion is satisfied $[20,21,22]$. In these cases, the calculation's answer is normally the 'pseudo surface' shape, an average membrane that would be obtained after the wrinkles have been removed from the mid-plane of the deformed geometry.

In the shell model representation, five degrees of freedom per node and bending stiffness are present. Therefore no additional models are needed, and wrinkles are naturally taken into account and reproduced.

In Fig.22 a mesh sensitivity study was performed for the four analysed meshes, and four sections on the deformed sail have been extracted. For all meshes wrinkles develop, but the wrinkle's wavelength decreases as the mesh gets finer. As wrinkling is a buckling related phenomenon, it is very difficult to get convergence in terms of wrinkle development. However, the sail's average deformation and the wrinkles wave length become coherent for all the three finer meshes, represented in the figure with continuous lines.

It is well known that the answer of a coarser mesh is generally stiffer, since the structure has less degrees of freedom. This is what happens in the lower section of the sail (section S4 in Fig.22) where the answer obtained with the coarser mesh tends to be stiffer. This is the region where wrinkles do not develop. In the higher part of the sail however, the extensive development of wrinkles causes the structure to be locally stiffer, resulting in smaller nodal displacements. This is confirmed by the comparison with the membrane analysis, performed in the last section. In the higher part of the sail, displacements calculated with shell elements are smaller, since wrinkles produce some additional stiffness. In the lower part of the sail, where wrinkles does not develop, the shell answer is significantly less stiff.

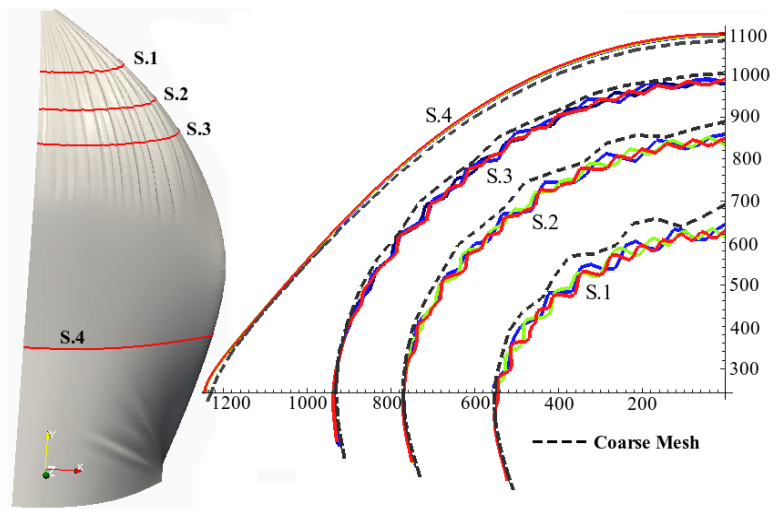

Fig.22 Wrinkling development

\section{EFFECT OF WRINKLES ON THE FLOW}

A coupling routine has not yet been established for the fluid structure interaction, and it will be the first priority in the future development of the research. However, some tests have been carried in the fluid domain, in order to investigate whether wrinkles can affect the air flow. This study can give useful guidelines in order to decide the best strategy for the coupling algorithm. The two dimensional sail section, already studied in the beginning of this paper, has been then modified introducing some oscillations around the base geometry. From the structural results presented in the previous section, it was estimated to introduce 12 oscillations with $\mathrm{D} / 12$ amplitude, where D is the sail section diameter, in the central part of the device. All relevant parameters remained unchanged, but due to the oscillation of the geometry an increase of about $8 \%$ in the area of the device can be estimated. Results are presented in Fig.24, where the drag force acting on the device is sketched over time. In examining the behaviour, quite a different time history can be observed.

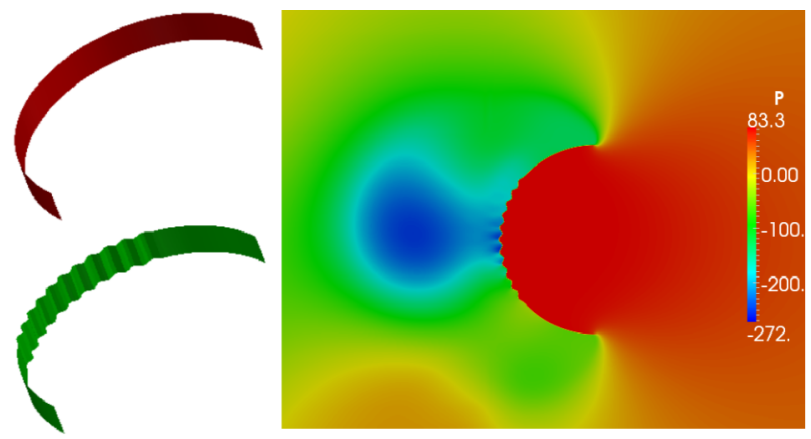

Fig.23 Effect of a wrinkled surface on vortex generation

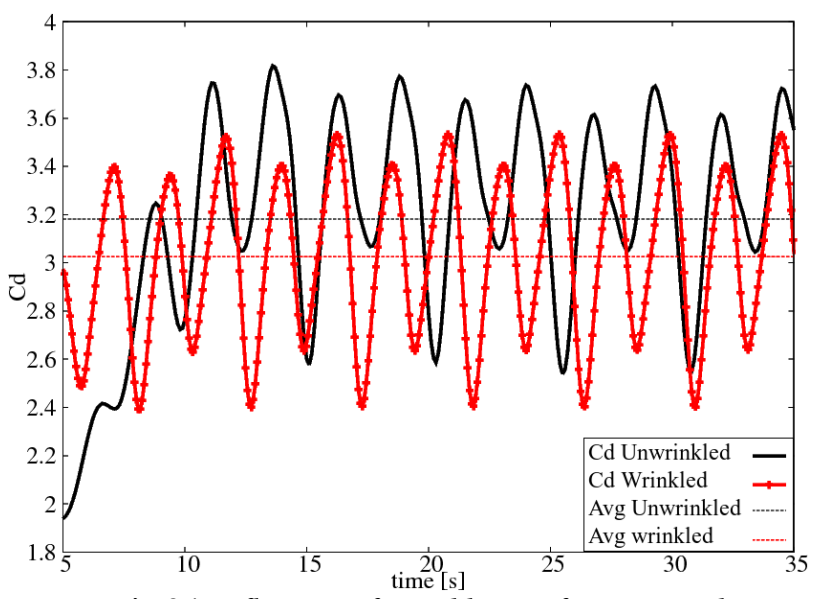

Fig.24 Influence of wrinkles on forces time history

Wrinkles seem in fact to reduce the influence of the lower pressure induced by the secondary vortex. This happens since the secondary recirculation zone, which would collapse back on the sail surface, is split into a series of smaller vortices (Fig.23), the influence of which is lower on the global force generation. By averaging the time signal in the periodic region of interest, the drag coefficient can be estimated as 3.246 for the unwrinkled 
case, and 3.092 for the wrinkled case, the increment is approximately $5 \%$.

Such results are not expected to maintain the same relative importance in the three-dimensional case, where the mixing of the flow is higher. Furthermore, the amplitude of wrinkles tends then to disappear when moving away from the higher part of the sail head. It is therefore not clear at the present stage if a wrinkled surface can significantly affect the sail force generation, globally or locally. Further studies will be undertaken in the near future.

\section{CONCLUSIONS}

Phenomena involved in sail fluid structure interactions have been here analysed, and an approach has been proposed, which involves dynamic structural calculations. The impact of unsteadiness on sail-type structures was investigated, and results seem to confirm that unsteady phenomena will influence the correct prediction of lift and drag. A new approach was proposed for modelling the structural fabric, using shell finite elements. With such elements all involved phenomena can be reproduced, thus overcoming the issue of defining a suitable additional model for taking into account sail buckling that results in wrinkles.

Much work will be needed for gaining confidence in the dynamic mesh handling. The example reported in the appendix will be used as a starting point for imposing multi dimensional rigid body motions as first, and then body deformations. When this is done, coupling between the structural and fluid codes will be performed, but the best strategy for doing this is still to be investigated.

For a structural perspective, the complexity of the model should be increased, considering reinforcements at the sail corners, and investigating the role of such discontinuity in the wrinkling generation. Furthermore, the role of damping will be investigated for understanding if it is necessary, and how it influences the calculation in the FSI coupling.

\section{REFERENCES}

1. MILGRAM, J., 'The analytical design of yacht sails', Transactions SNAME 76, 1968.

2. KREBBER, B. and HOCHKIRCH, K., 'Numerical investigation on the effects of trim for a yacht rig', High Performance Yacht Design Conference, Auckland, 2006.

3. SCHNEIDER A., ARNONE, A., SAVELLI, A., BALLICA, A. and SCUTELLARO, P., 'On the Use of CFD to Assist with Sail Design', The 16th Chesapeake sailing Yacht Symposium, Annapolis, Maryland, 2003.

4. MALPEDE, S. and BARALDI, A., 'A fully integrated method for optimizing fiber-membrane sails', High Performance Yacht Design Conference, Auckland, 2008.
5. MOUNOURY, S., HAUVILlE, F., DURAND, M., ASTOLFI, J. and ROUX, Y., 'Nonlinear aero-elasticity applied on sails', 10èmes journées de l'hydrodynamique, Nantes, 2005.

6. VERNENGO, G. and BRIZZOLARA, S., 'Application of a vortex lattice method to the analysis of sail plans in upwind condition', 13th Congress of International Maritime Association of Mediterranean, Istanbul, 2009.

7. CHAPIN, V.G., DULLIAND, G and CHASSAING, P., 'Design optimization of interacting sails through viscous CFD', Innov'Sail, Lorient, 2008.

8. QUERARD, A. and WLSON, P., 'Aerodynamic of modern square head sails: a comparative study between wind-tunnel experiments and RANS simulations', The modern yacht conference, Southampton, 2007.

9. RENZSCH, H., MULLER, O. and GRAF, K., 'FlexSail - A fluid structure interaction program for the investigation of Spinnakers', High Performance Yacht Design Conference, Auckland, 2008.

10. PRUKL, P., 'The CST - An insidious survivor from the infancy of FEA', 11th Symposium on Finite Element Methods in South Africa, 1992.

11. BATHE, K.J., 'Finite element procedures', Prentice Hall, 1996.

12.CHAPELLE, D. and BATHE, K.J., 'The finite element analysis of Shells - fundamentals', Springer, 2003.

13.ANDERSON, J., 'Computational Fluid Dynamics The basis with applications', McGraw-Hill, 1995.

14. FERZIGER, J. and PERIC, M., 'Computational Methods for Fluid Dynamics’, Springer, 1999.

15. COLLIE, S.J., GERRITSEN, M. and JACKSON, P., 'A review of turbulence modelling for use in sail flow analysis', University of Auckland, School of Engineering, Report No. 603, 2001

16.PATTENDEN, R., BRESSLOFF, N., TURNOCK, S. and ZHANG, X., 'Unsteady simulations of the flow around a short surface-mounted cylinder', Int. J. Numer. Meth. Fluids 53, 2006.

17. COLLIE, S.J., JACKSON, P., GERRITSEN, M. and FALLOW, J., 'Two-dimensional CFD based parametric analysis of downwind sail design', University of Auckland, School of Engineering, 2003

18. LI, J. and CHAN, S., 'An integrated analysis of membrane structures with flexible supporting frames', Finite Elements in Analysis and Design 40, 2004. 
19. TRIMARCHI, D. and RIZZO, C., 'A FEM-Matlab code for Fluid-Structure interaction coupling with application to sail aerodynamics of yachts', 13th Congress of International Maritime Association of Mediterranean, Istanbul, 2009.

20. KANG, S. and IM, S., 'Finite Element analysis of wrinkling membranes', Transactions ASME, 1997.

21. LEE, E. and YOUN, S.,' Finite element analysis of wrinkling membrane structures with large deformations', Finite Elements in Analysis and Design 42, 2006.

22. PATON, J. and HEPPEL, P., 'Fluid Structure Interaction of yacht sail', Innov'Sail, Lorient, 2008.

23. DONEA, J., HUERTA, A., PONTHOT, J. and RODRIGUEZ-FERRAN, A., 'Arbitrary LagrangianEulerian Methods', Enciclopedia of Computational Mechanics - Ch.14, 2004.

\section{APPENDIX: A MOVING MESH STUDY}

An Arbitrary Lagrangian Eulerian (ALE) approach should be chosen for performing unsteady FSI coupling. Using this approach it is possible to couple the structural calculation, performed on a Lagrangian grid, and the fluid calculation, which uses an Eulerian grid. General informations about ALE coupling can be found in [23]. The general principle is that the fluid grid should follow the structural grid on the fluid-structure interface, remain unchanged at the fluid boundaries and 'arbitrarily' deform in the rest of the domain. 'Arbitrarily' means in this context that any deformation rule can be chosen. In general the inner mesh is updated solving a spring analogy, a Laplacian, or some more complex continuum mechanics analogies. In any case, when dealing with the mesh motion the fluid equations should be modified, in order to take into account the mesh deformation velocity, thus maintaining a consistent system. OpenFOAM offers a module for dynamic mesh handling, called pimpleDyMFOAM. This module incorporates many mesh deformation solver, and can deal with both laminar and turbulent flows. Preliminary tests have been performed on a simplified geometry, where velocities are imposed to a rigid vertical beam moving in a fluid domain. Since no velocity is imposed to the flow, the fluid velocity field derives entirely from the beam motions. In Fig.25, a sequence of such dynamic mesh handling is presented. A rigid bar starts moving with constant velocity $\left(1 \mathrm{~m} \mathrm{~s}^{-1}\right)$. After six second the bar rests, and a negative velocity is then applied. The colourmap shows the velocity magnitude, and it is possible to see the complex vortex generation due to the rigid body motion.
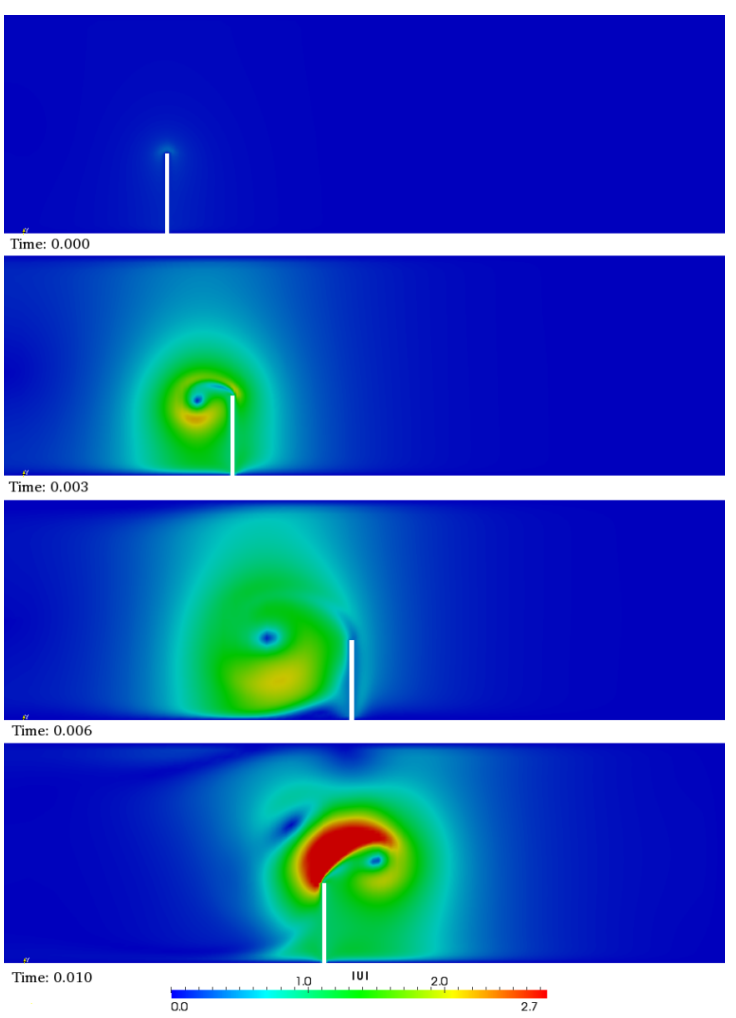

Fig.25 Dynamic mesh handling

\section{AUTHORS BIOGRAPHY}

Daniele Trimarchi is a $\mathrm{PhD}$ student in the University if Southampton. His research field is fluid structure interactions applied to yacht sails. His previous experience includes the development of a Matlab finite element code dealing with membrane element.

Dr. Stephen Turnock is Reader in Maritime Fluid Dynamics and heads the Performance Sports Engineering Laboratory. His research focuses on the synthesis of computational, experimental and analytical fluid dynamics applied to maritime design.

Dr. Dominic Taunton is a Roberts Fellow in Hydro sciences in the School of Engineering Sciences and member of the Performance Sports Engineering Laboratory. His research interests include experimental hydrodynamics and human factors for high-speed craft.

Dr. Dominique Chapelle is the head of INRIA-MACS team. His research field is mathematical models used in the structural mechanics, and in particular shell finite element models. The main application for such models is in biomathematics, namely in the simulation of the heart. 